\title{
Multipolar third-harmonic generation in fishnet metamaterials
}

\section{Supporting Information}

\section{Experimental retrieval of the effective parameters.}

We experimentally measure the transmission and reflection phase (Fig. S1, dots) in the spectral region of the pump laser. Combined with the measured transmission and reflection intensities (Fig. S1, curves) we can calculate the complex transmission and reflection. For the phase measurements, we employ interferometry techniques [1]. Specifically, for measuring the phase in transmission, we use the Mach-Zehnder interferometer, while for the measurements of a phase in reflection we employ the Michelson-Morley interferometer. We experimentally detect the interference pattern of the sample and the reference waves, coming at an angle with respect to each other. We use a single-image technique to retrieve the phase information. Specifically, we perform a Fourier-transform of the image of the interference pattern and in Fourier-image we filter-out all the spatial frequencies except the frequencies corresponding to a single maximum of the first order. After the filtering, we perform the inverse Fourier-transform, which gives us a two-dimensional distribution (image) of the complex field, where the phase of the field represents a phase difference between the sample and reference beams.

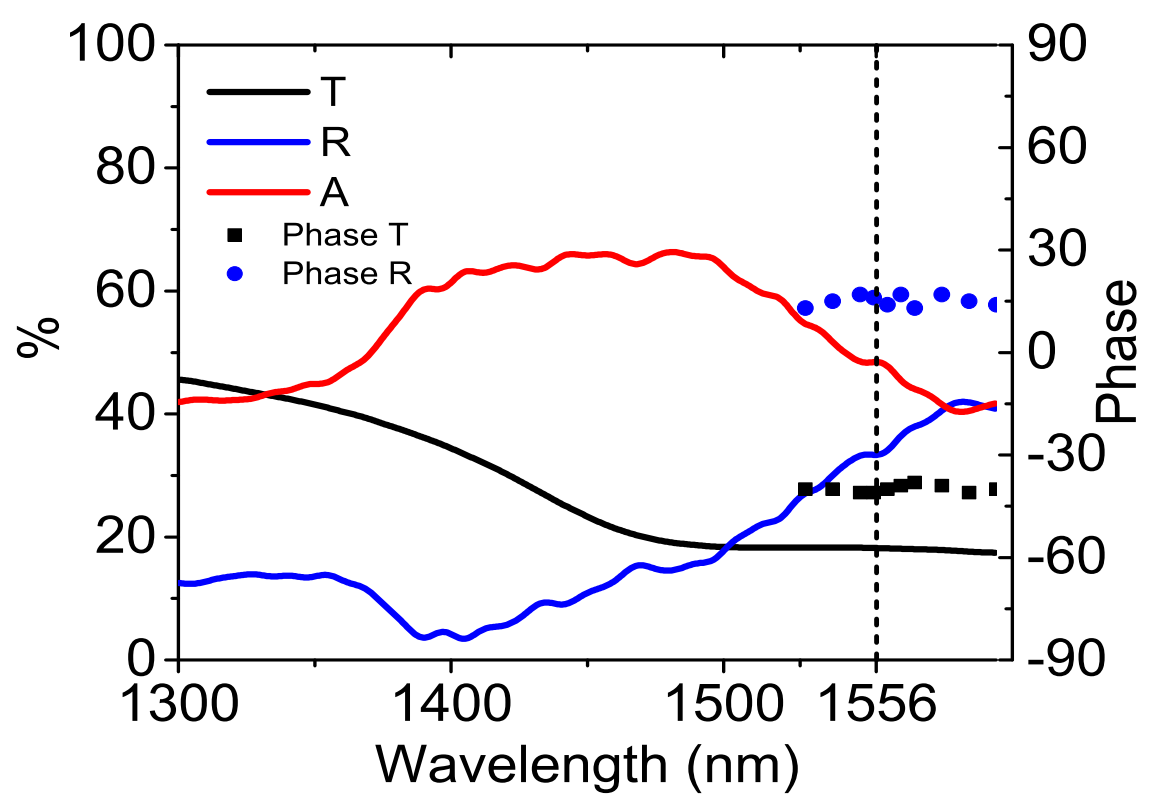

Figure. S1. Experimentally measured transmission (black) and reflection (blue) spectra (lines). Calculated absorption (red line). Phase (dots) spectra of the fishnet metamaterial.

We use inverted Fresnel equations to extract the effective refractive index and surface polarizabilities of the structure. The results are shown in Fig. S3 and the effective parameters of the fishnet metamaterial at the pump wavelength are shown in Table. 1. 


\begin{tabular}{|l|l|}
\hline $\mathbf{n}$ (refractive index) & $-0.62+\mathrm{i} 5.98$ \\
\hline $\boldsymbol{\alpha}^{\mathrm{e}}$ (surface electric polarizability) & $(1.58+\mathrm{i} 5.99) 10^{-16}$ \\
\hline $\boldsymbol{\alpha}^{\mathrm{m}}$ (surface magnetic polarizability) & $(-10.3+\mathrm{i} 18.6) 10^{-16}$ \\
\hline
\end{tabular}

Table 1. Effective fishnet parameters at $1556 \mathrm{~nm}$ pump wavelength.

\section{Theoretical calculations of the effective parameters.}

We use Lumericals FDTD Solutions to simulate the spectra of the fishnet metamaterial. As shown in Fig. S2. The black dashed line represents the location of the pump's wavelength.

In Fig. S3 we compare the calculated effective refractive index $n$, where the real part of $n$ is plotted as the blue curve. We observe that the refractive index is negative in the spectral range of our pump laser, indicating strong magnetic response of the structure.

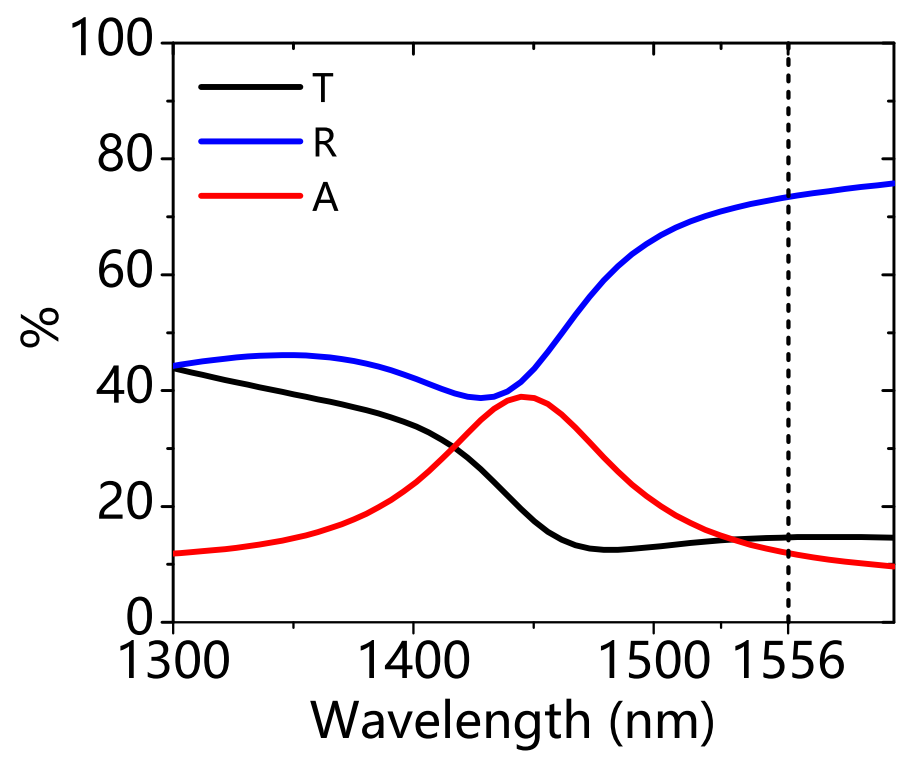

Figure S2. Simulated linear spectrum of the fishnet metamaterial.

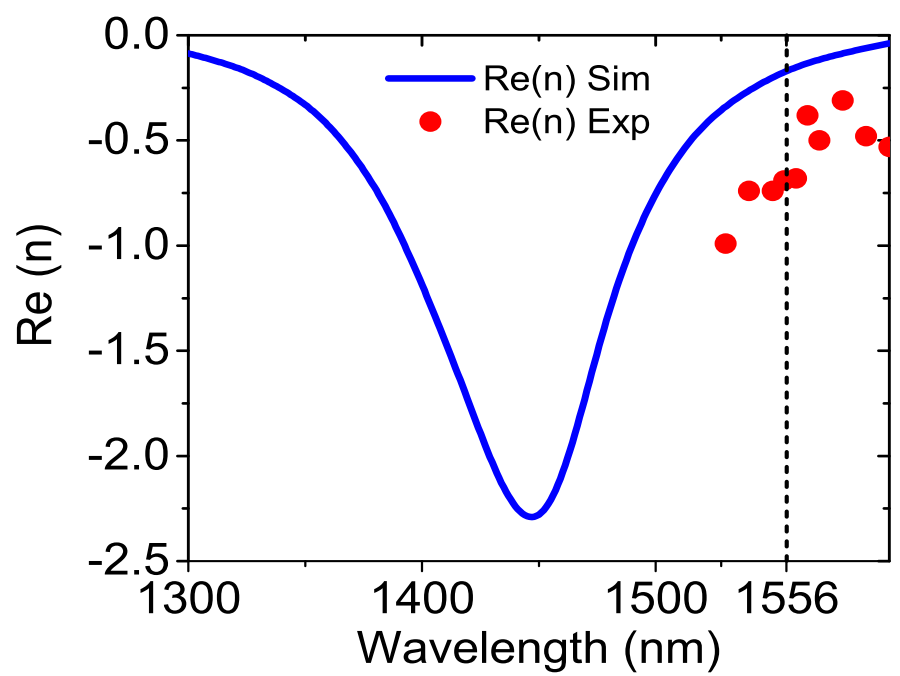

Figure S3. Comparison of effective refractive index, real part. Blue line-calculated values; red dotsexperimentally obtained values. 

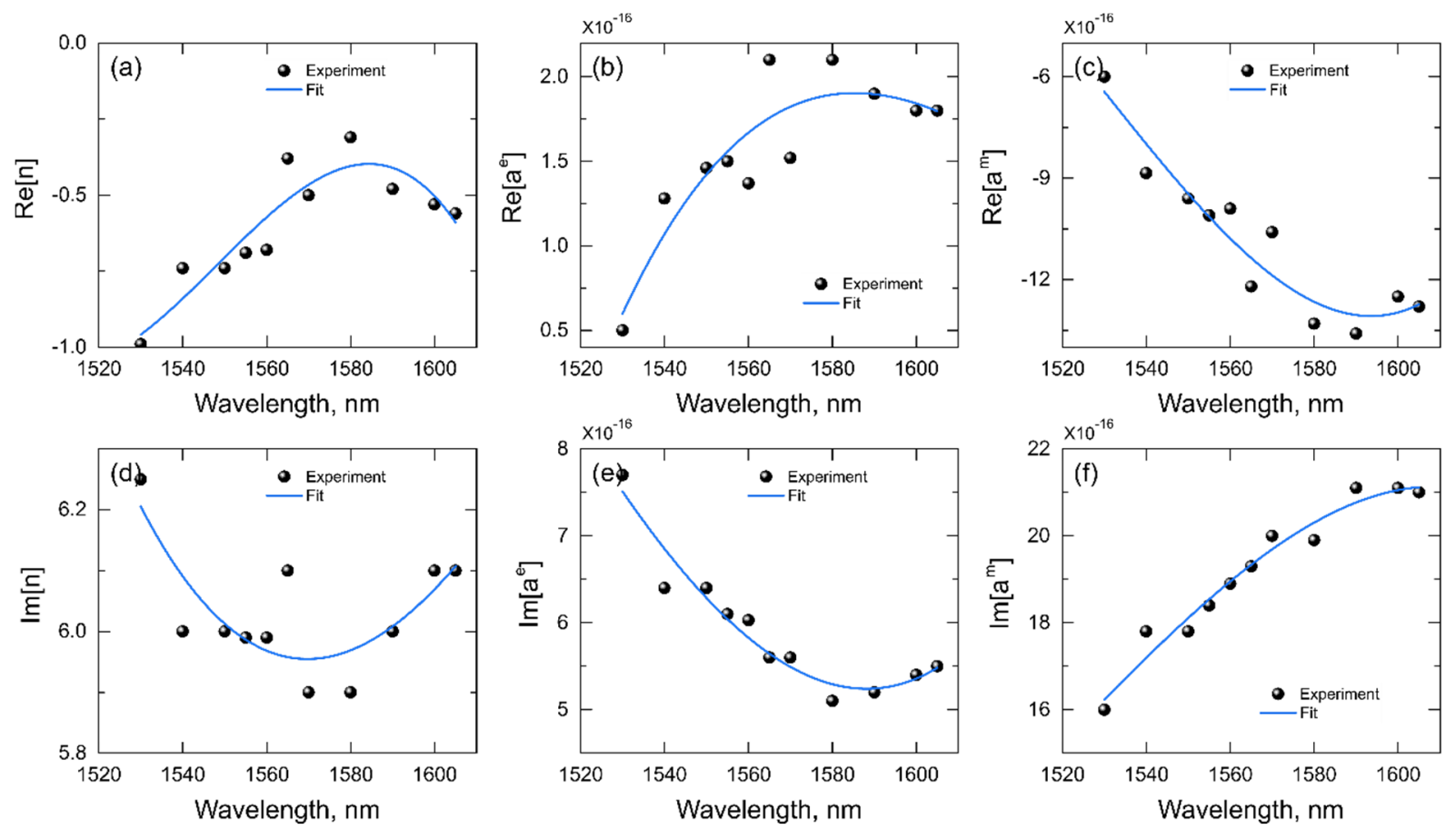

Figure. S4. Experimentally extracted effective parameters of the fishnet metamaterial: real parts $(a-c)$ and imaginary parts $(d-f)$ of the effective refractive index $(a, d)$, the surface electric polarizability $(b, e)$ and the surface magnetic polarizability $(c, f)$.

\section{Multipolar interference of near-neighbour unit cells.}

In our experiments the metamaterial unit cell size is $500 \mathrm{~nm}$, while the diffraction-limited pump beam spot size is over $2000 \mathrm{~nm}$ large, i.e. the pump beam illuminates several unit cells.

To take this into account in our multipolar expansion we consider interference of the radiation patterns of several near-neighbour unit cells. In Fig. S5 we show examples of the cases when of 4, 5 or 12 neighbouring unit cells are excited (see Fig. S5). For all the cases we use the same set of complex amplitudes $C_{1}, C_{2}$ and $C_{3}$. We find that qualitatively the radiation patterns for all the cases look similar when at least 4 unit cells are taken into account, however the 12 unit-cell case gives the best quantitative match to our experiments. 
(a)

(b)
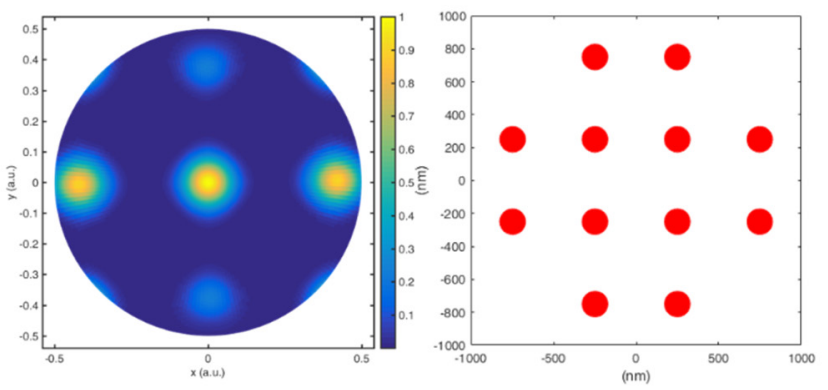

(c)
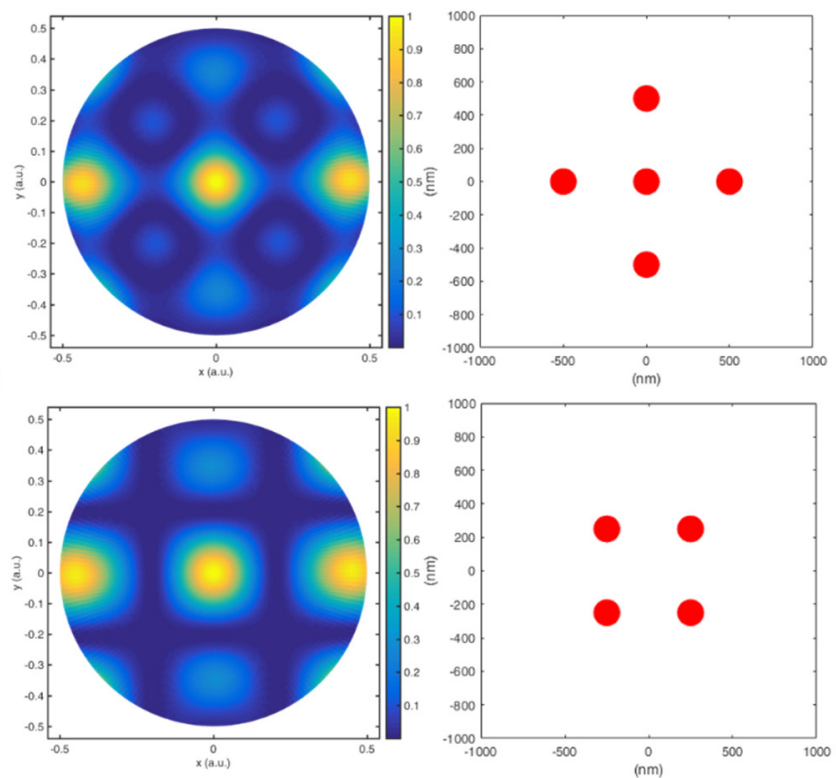

Figure S5. Comparison of the multipole emitter array types. (a) Red dots on the right picture represent the array locations of the multipole emitters. It considers only the 12 fishnet unit cells within the Gaussian beam waist of the pump's focal spot. The calculated back focal plane image is derived from the superposition of these multipole emitters in this array. (b) The calculated back focal plane image of multipole model. The same complex amplitudes of ED, $M D$ and $E Q$ is used but only 5 emitters are included in the array. The general radiation features are the same as that in (a). (c) The case with 4 emitters.

\section{References}

[1] Kruk, S. S.; Wong, Z. J.; Pshenay-Severin, E.; O'Brien, K.; Neshev, D. N.; Kivshar, Y. S.; Zhang, X. Nat Commun. 2016, 7. 